\title{
Técnicas e Táticas no Tratamento Endovascular do Aneurisma da Artéria Renal
}

\author{
Keillyanne Jaira Ferreira Barros ${ }^{1}$, Patrick Bastos Metzger ${ }^{1}$, Fabio Henrique Rossi ${ }^{1}$, \\ Thiago Osawa Rodrigues ${ }^{1}$, Samuel Martins Moreira' ${ }^{1}$, Ana Claudia Gomes Petisco ${ }^{2}$, \\ Nilo Mitsuru Izukawa' ${ }^{1}$ Antonio M. Kambara' ${ }^{1}$
}

\section{RESUMO}

Introdução: Os aneurismas da artéria renal são raros e constituem um desafio ao tratamento endovascular. Nosso objetivo foi descrever e analisar as técnicas e táticas no tratamento endovascular do aneurisma da artéria renal, verificando os resultados a curto e médio prazos de uma série consecutiva de casos. Métodos: Estudo retrospectivo, de procedimentos realizados no período de janeiro de 2010 a dezembro de 2013, em que foram analisados: o sucesso técnico e terapêutico, a morbimortalidade, e a taxa de vazamentos e de reintervenções. Resultados: Em um total de seis pacientes tratados, a idade média foi de $41 \pm 5$ anos e todos eram do sexo feminino. A maioria apresentou aneurismas saculares tipo II (83,3\%). Foram utilizadas técnicas de remodelamento com uso de stent e molas em quatro casos, embolização segmentar renal em um caso e tratamento com endoprótese Multilayer $^{\circledR}$ em outro. O sucesso técnico e terapêutico foi de 100 e 83,3\%, respectivamente. Em um paciente, houve isquemia de polo superior renal, que evoluiu para hematúria e dor incontrolável, necessitando de nefrectomia. Não ocorreram óbitos e nem oclusão das artéria renais nativas e de seus ramos durante o acompanhamento de 1 ano. Conclusões: $O$ tratamento endovascular do aneurisma de artéria renal demonstrou ser uma alternativa viável à cirurgia convencional com baixa morbidade. $\mathrm{O}$ estudo detalhado da vascularização renal e da localização do aneurisma determina a escolha da técnica endovascular a ser utilizada. O aneurisma da artéria renal do tipo II foi a morfologia mais frequentemente encontrada e pode ser tratado com sucesso por técnicas de remodelamento com o uso de stent e mola.

DESCRITORES: Aneurisma. Artéria renal. Embolização terapêutica. Procedimentos endovasculares.

\section{ABSTRACT}

Techniques and Strategies for the Endovascular Treatment of Renal Artery Aneurysm

Background: Renal artery aneurysms are rare and constitute a challenge to endovascular treatment. Our objective was to describe and analyze the techniques and strategies for the endovascular treatment of renal artery aneurysms verifying short and medium-term results in a consecutive series of cases. Methods: Retrospective study of procedures performed from January 2010 to December 2013, analyzing technical and therapeutic success, morbidity and mortality, the rate of endoleaks and reinterventions. Results: In a total of six patients treated, mean age was $41 \pm 5$ years and all patients were female. The majority of the patients had type 2 saccular aneurysms (83.3\%). Remodeling techniques using stent and coils were used in four cases, embolization of renal polar branch was used in one case and treatment with a Multilayer ${ }^{\circledast}$ endoprosthesis in another case. Technical and therapeutic success rates were 100\% and $83.3 \%$, respectively. In one patient there was upper renal pole ischemia, which progressed to uncontrollable hematuria and pain, requiring nephrectomy. There were no deaths or occlusion of the native renal artery and its branches during the 1-year follow-up. Conclusions: Endovascular treatment of renal artery aneurysm proved to be a feasible alternative to conventional surgery with low morbidity. A detailed study of renal vasculature and aneurysm location determines the choice of the endovascular technique to be used. Type II renal artery aneurysm was the most frequent morphology observed and may be successfully treated by remodeling techniques using stents and coils.

DESCRIPTORS: Aneurysm. Renal artery. Embolization, therapeutic. Endovascular procedures.

\footnotetext{
1 Centro de Intervenções Endovasculares, Instituto Dante Pazzanese de Cardiologia, São Paulo, SP, Brasil.

2 Seção Médica de Ecografia Vascular, Instituto Dante Pazzanese de Cardiologia, São Paulo, SP, Brasil.

Correspondência: Patrick Bastos Metzger. Avenida Dr. Dante Pazzanese, Setor de Hemodinâmica, 500 - Vila Mariana - CEP: 04012-909 - São Paulo, SP, Brasil

E-mail: patrickvascular@gmail.com

Recebido em: 3/1/2014 • Aceito em: 3/3/2014
} 
0 aneurisma da artéria renal (AAR) é um evento raro, cuja prevalência é de $0,01 \% .^{1,2} \mathrm{Na}$ última década, com o uso de métodos diagnósticos não invasivos, como a ultrassonografia Doppler (USG-D) e a angiotomografia computadorizada (TC), o número de casos diagnosticados vem aumentando. ${ }^{3}$

Embora a história natural dos AAR seja pouco conhecida, e os sintomas sejam inespecíficos ou inexistentes, complicações potenciais, como embolização e ruptura, são relatadas em 5 a 10\% dos casos, levando a uma taxa de mortalidade elevada, especialmente em mulheres grávidas ou em pacientes com poliarterite nodosa (PAN). ${ }^{4,5}$ Dessa maneira, as indicações para o tratamento incluem pacientes sintomáticos, mulheres gestantes ou em idade fértil, e pacientes assintomáticos com aneurismas $>2 \mathrm{~cm}$ ou com PAN. ${ }^{3,6}$

Tanto o tratamento cirúrgico convencional como o autotransplante renal apresentam taxa de mortalidade variando de zero a 4\%, com taxa de complicação de 10 a $30 \% .^{7,8}$ Nos últimos anos, com o progresso do tratamento endovascular e o desenvolvimento de novos dispositivos, os AARs têm sido abordados com menor morbimortalidade e com a preservação da árvore vascular nativa. ${ }^{9}$

O objetivo deste estudo foi descrever e analisar as técnicas e táticas do tratamento endovascular dos AAR, avaliando o sucesso técnico e terapêutico, a morbimortalidade, e a taxa de vazamentos e de reintervenções de uma série consecutiva de pacientes, acompanhados por 1 ano.

\section{MÉTODOS}

\section{Características do estudo}

Trata-se de um estudo retrospectivo, longitudinal, observacional, realizado em um centro de referência para patologias cardiovasculares. Foram critérios de inclusão no estudo: pacientes de ambos os sexos, com AAR sintomáticos, AAR com diâmetro $>2 \mathrm{~cm}, \mathrm{AAR}$ em mulheres gestantes ou em idade fértil e AAR associados à PAN. Foram excluídos pacientes com creatinina sérica $>2,0 \mathrm{mg} / \mathrm{dL}$ ou clearence de creatinina $<30 \mathrm{~mL} / \mathrm{min}$.

A programação terapêutica foi realizada com TC em todos os casos, sendo a arteriografia pré-operatória um método diagnóstico opcional. Todas as tomografias foram reconstruídas no software OsiriX ${ }^{\circledR}$ MD em modos tridimensional e de reconstrução multiplanar (Figura 1).

\section{Planejamento endovascular}

Com o estudo angiotomográfico e/ou arteriográfico prévio, foram observadas: a morfologia do aneurisma (sacular ou fusiforme), a presença de ramos de drenagem, a extensão do colo aneurismático, o acometimento de zonas de bifurcação, e a distância da origem da artéria renal ao aneurisma e do aneurisma até a bifurcação da artéria renal.
O planejamento do tratamento endovascular dos AARs foi realizado de acordo com sua classificação topográfica ${ }^{10}$ em tipos I (aneurismas localizados na artéria renal principal), II (aneurismas localizados no hilo) e III (aneurismas intrarrenais) (Figura 2).

\section{Técnicas e táticas para aneurisma renais tipo I}

a) Endoprótese GORE ${ }^{\circledR}$ VIABAHN $^{\circledR}$ (W.L. Gore \& Associates Inc., Flagstaff, Estados Unidos)

É uma opção de tratamento no AAR tipo I localizados até $15 \mathrm{~mm}$ de distância do óstio da artéria renal ou do hilo, sem ramos nutridores oriundos do saco aneurismático. Quando esses limites não são respeitados, existe risco de vazamentos e perpetuação do fluxo dentro do saco aneurismático, com consequente insucesso terapêutico.

Não deve ser utilizado em AAR tipo II pelo risco de cobrir ramos renais importantes, nem nos AAR tipo III, devido a seu alto perfil e pouca flexibilidade para navegar em vasos distais finos, além de apresentar risco de tromboembolismo ou trombose, quando utilizado nesses cenários.

b) Embolização seletiva com mola

Pode ser usada nos aneurismas dos três tipos, sendo que sua melhor indicação são nos saculares com colos $<4 \mathrm{~mm}$ ou na relação diâmetro do aneurisma/colo $>2: 1 .{ }^{9}$ Nesses casos, são necessárias técnicas que utilizam microcateteres para cateterização superseletiva do aneurisma, além de micromolas com perfil de 0,018. A preferência dos autores é pelas molas AZUR ${ }^{\circledR}$ (Terumo Interventional Systems, Somerset, Estados Unidos) e pelo microcateter Progeat ${ }^{\circledR}$ 2,4 F (Terumo Interventional Systems, Somerset, Estados Unidos). Um ponto importante é que o tamanho da primeira mola deve ser igual ao do saco aneurismático, para possibilitar uma configuração circular dentro do aneurisma.

Essa técnica não deve ser utilizada em aneurismas complexos, aneurismas com colos largos ou quando ramos arteriais saem do saco aneurismático. Nesses casos, recomendam-se as técnicas de remodelamento.

\section{Técnicas e táticas para aneurismas renais tipo II}

a) Endoprótese multicamadas ou Multilayer ${ }^{\circledR}$ (Cardiatis, Isnes, Bélgica)

A endoprótese Multilayer ${ }^{\circledR}$ é usada principalmente para o tratamento de aneurismas periféricos, porém, em estudos recentes, seu uso tem sido relatado em aneurismas renais. A estrutura da endoprótese, em várias camadas entrelaçadas e juntas, cria uma configuração em múltiplos planos, que age lentificando o fluxo turbulento dentro do saco aneurismático, o que contribui para sua trombose, enquanto melhora o fluxo laminar na artéria principal e seus ramos. ${ }^{11,12}$ 


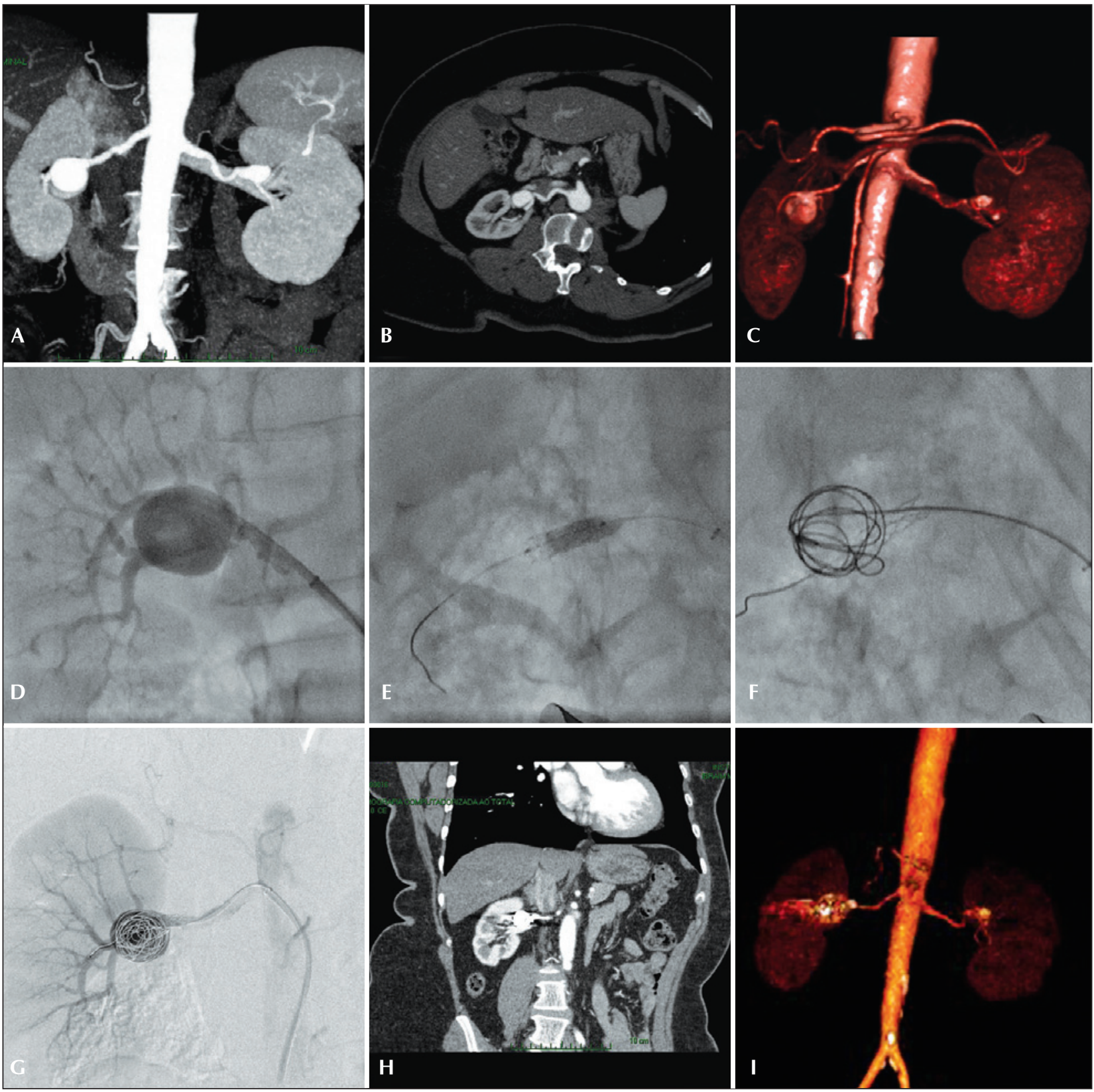

Figura 1. Planejamento pré-operatório, tratamento endovascular e acompanhamento pós-operatório do aneurisma renal tipo II. (A e B) Angiotomografia em cortes coronal e axial. (C) Reconstrução volumétrica do aneurisma de artéria renal. (D) Arteriografia renal. (E) Implante de stent expansível por balão em colo do aneurisma renal. (F) Liberação de molas em saco aneurismático após a cateterização do mesmo entre as malhas do stent. (G) Arteriografia final, sem vazamentos ou oclusão de ramos renais. (H) Angiotomografia pós-operatória em corte coronal. (I) Reconstrução volumétrica de angiotomografia pós-operatória.

A pouca flexibilidade e seu alto perfil limitam seu uso em aneurismas do tipo III.

b) Técnicas de remodelamento: stent e molas

Essa técnica é uma importante ferramenta no tratamento dos aneurismas com colos aneurismáticos largos dos tipos I e II. A utilização de stent expansível por balão funciona como suporte para a liberação das micromolas dentro do saco aneurismático, após a cateterização seletiva com cateteres diagnósticos $4 \mathrm{~F}$ ou microcateteres entre as malhas do stent (Figura 3). Com isso, ocorre uma alteração dos parâmetros hemodinâmicos locais, com redirecionamento do fluxo para a artéria nativa e endotelização posterior das malhas do stent. Os autores têm preferência pelo uso do stent expansível por balão Palmaz ${ }^{\circledR}$ Blue $^{\circledR}$ (Cordis Corporation, Warren, Estados Unidos) associado ao uso de molas com perfil de 0,018 AZUR $^{\circledR}$ (Terumo Interventional Systems, Somerset, Estados Unidos). 


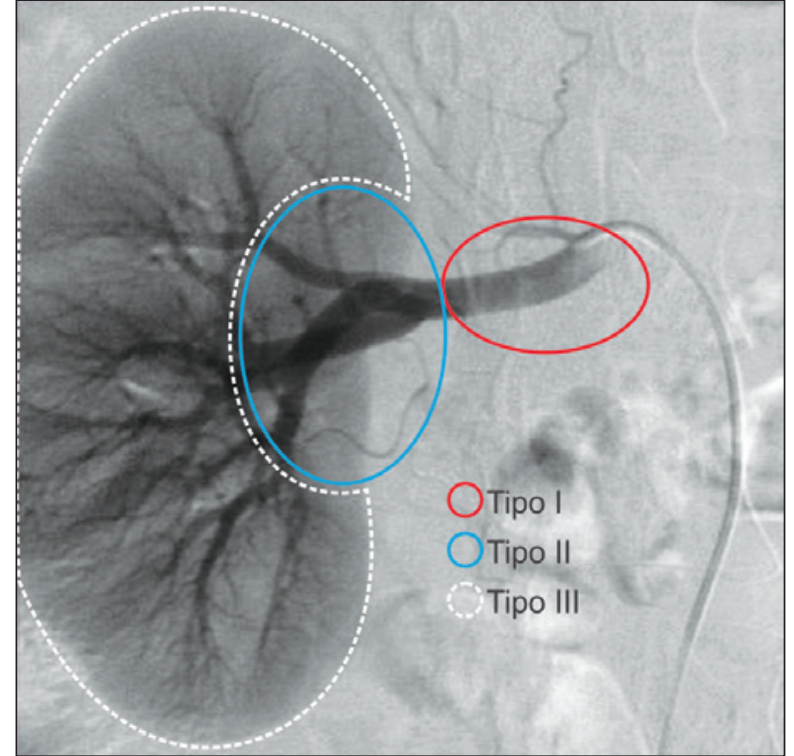

Figura 2. Classificação dos tipos de aneurisma de artéria renal segundo sua localização. Tipo I: aneurismas localizados na artéria renal principal; tipo II: aneurismas localizados na região do hilo renal, após a bifurcação arterial da artéria renal principal; tipo III: aneurismas de artéria renal intraparenquimatosos.

\section{Técnicas e táticas para aneurismas renais tipo III}

a) Embolização superseletiva de ramo segmentar renal com uso de N-butil cianoacrilato Histoacryl ${ }^{\circledR}$ (B Braun, Tuttlingen, Alemanha)

O Histoacryl ${ }^{\circledR}$ é um adesivo tecidual que, quando em contato com fluidos iônicos, como o sangue, solidifica-se, resultando na oclusão do vaso. A cola é usada em uma mistura com Lipiodol ${ }^{\circledR}$ (Guerbet, Anlney-Sous-Bois, França), para que se torne visível ao raio $X$ e retarde sua polimerização. Nós usamos uma concentração em torno de 30 a $50 \%$ de cola na solução.

Essa técnica é utilizada para aneurismas tipo III, em que a oclusão do vaso segmentar nativo provoca uma pequena área de infarto renal. Para a realização dessa técnica, é necessária a utilização de microcateteres para micronavegação cuidadosa dos vasos segmentares renais.

\section{Procedimento}

Todos os procedimentos foram realizados no laboratório de Hemodinâmica do Centro de Intervenções Endovasculares ou em sala híbrida no Instituto Dante Pazzanese de Cardiologia.

Os pacientes foram tratados sob anestesia local. A profilaxia antimicrobiana foi realizada com 1,5 g de cefuroxima, no momento da indução anestésica. A abordagem foi preferencialmente realizada pela artéria femoral comum, por punção unilateral. Na impossibilidade dessa via de acesso, ou quando havia tortuosidade importante da artéria renal a ser cateterizada, optou-se pela abordagem da artéria braquial esquerda.

O controle radiográfico foi feito com aparelho Siemens ${ }^{\circledR}$ Artis Flat Panel, ou, na sala híbrida, com o aparelho Siemens ${ }^{\circledR}$ Artis Zeego Hybrid.

A arteriografia intraoperatória foi realizada em todos os pacientes. O pós-operatório imediato foi realizado na enfermaria em todos os casos.

\section{Acompanhamento pós-operatório}

Os pacientes foram acompanhados com avaliação ambulatorial aos 15, 30, 180, e 360 dias após o procedimento e, a seguir, anualmente. O controle com TC foi realizado no $30^{\circ}$ dia de seguimento ambulatorial. $\mathrm{O}$ USG-D foi realizado em 30 e 180 dias, e anualmente, com a finalidade de avaliar a presença de fluxo no saco aneurismático.

\section{Desfechos e definições}

O sucesso técnico foi definido quando a técnica escolhida para o tratamento do AAR foi realizada de forma previamente planejada. O sucesso terapêutico foi considerado quando a técnica escolhida para o tratamento do AAR foi realizada de forma previamente planejada, sem a presença de vazamentos ou de outras complicações que influenciassem o selamento adequado do aneurisma. A perviedade da artéria renal foi definida como obtenção da patência da artéria renal tratada, sem sinais de isquemia do parênquima renal correspondente.

Foram avaliadas, em 1 ano, a morbimortalidade e as taxas de vazamentos e de reintervenções em 1 ano.

\section{RESULTADOS}

No período compreendido entre janeiro de 2010 a dezembro de 2013, foram realizadas correções endovasculares de seis AAR consecutivos. Características demográficas, comorbidades e indicações de tratamento estão descritas na tabela 1. A média de idades foi de 41 \pm 5 anos. Todos os pacientes eram do sexo feminino e $83,3 \%$ eram assintomáticos, tendo sido diagnosticados casualmente em exames de imagem. A hipertensão arterial foi a comorbidade mais prevalente. Encontramos elevada incidência de fibrodisplasia (83,3\%), e não houve nenhum caso de PAN. Aneurisma $>2 \mathrm{~cm}$ em mulheres em idade fértil foi a principal indicação para o tratamento endovascular do AAR. Todos os pacientes foram tratados de forma eletiva. A via de acesso femoral foi usada em cinco casos (83,3\%), e o acesso braquial no caso restante.

O sucesso técnico foi de $100 \%$ e o sucesso terapêutico foi de $83,3 \%(n=5)$. O tempo médio de procedimento endovascular foi de 40 minutos (variação de 34 a 61 minutos), e o tempo médio de internação foi de 5 dias (variação de 2 a 15 dias). 

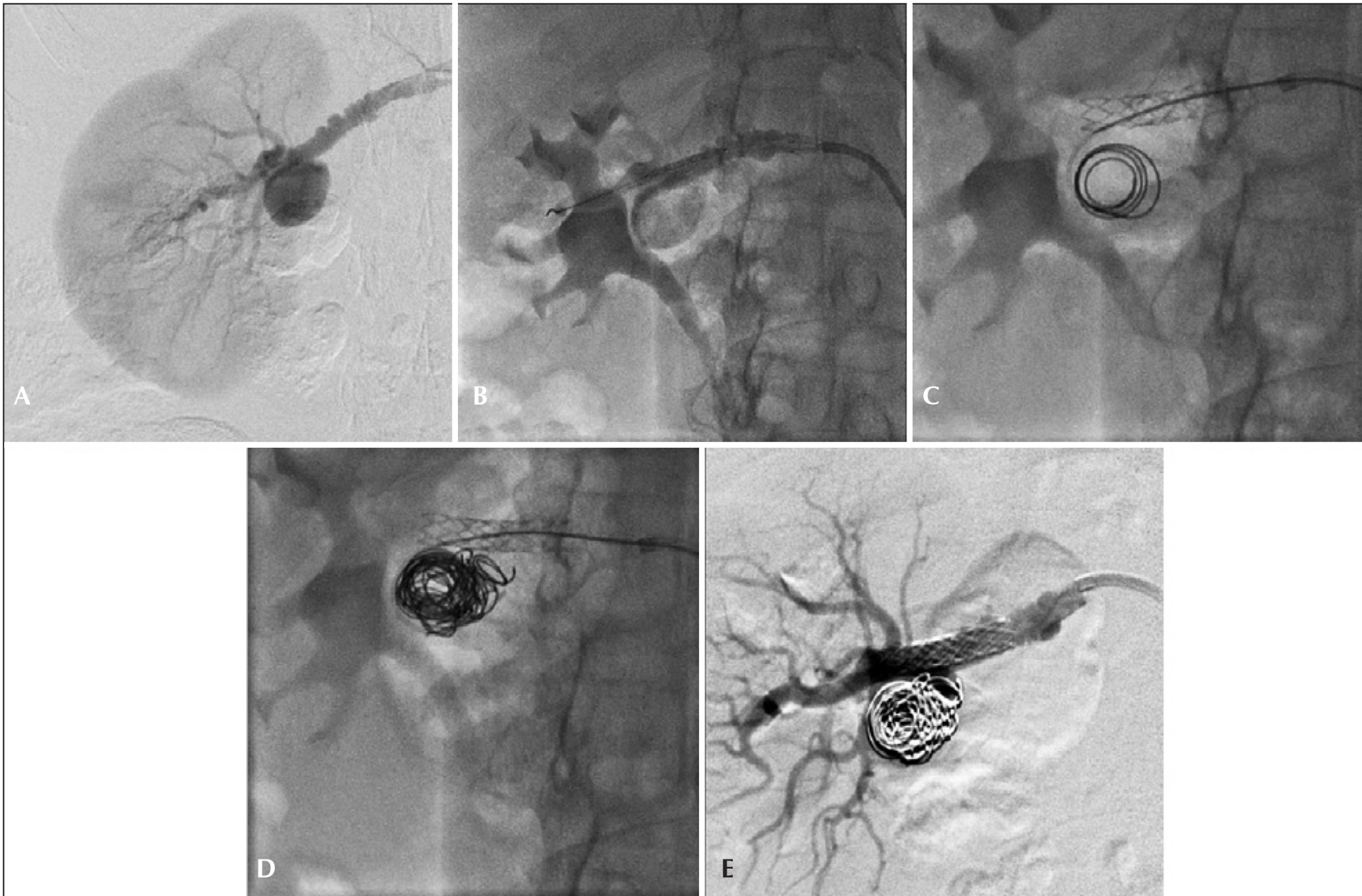

Figura 3. Passo a passo da técnica de remodelamento para tratamento de aneurisma de artéria renal tipo II com o uso de microcateter. (A) Arteriografia demonstrando aneurisma tipo II e doença fibrodisplásica associada. (B) Posicionamento e liberação de stent expansível por balão em colo de aneurisma renal. (C) Implante de micromolas 0,018 após micronavegação com microcateter 2,4 F entre as malhas do stent. (D) Aspecto final do adensamento de molas no saco aneurismático. (E) Arteriografia final sem vazamentos ou oclusões dos vasos nativos.

TABELA 1

Características clínicas e angiográficas

\begin{tabular}{lc}
\hline Características & $\mathbf{n = 6}$ \\
\hline Idade média, anos & $41 \pm 5$ \\
Sexo feminino, $\mathrm{n}(\%)$ & $6(100)$ \\
Doença sintomática, $\mathrm{n}(\%)$ & $1(16,6)$ \\
Diabetes mellitus, $\mathrm{n}(\%)$ & 0 \\
Hipertensão, $\mathrm{n}(\%)$ & $5(83,3)$ \\
Dislipidemia, $\mathrm{n}(\%)$ & $2(33,4)$ \\
Tabagismo, $\mathrm{n}(\%)$ & $2(33,4)$ \\
Cardiopatia isquêmica, $\mathrm{n}(\%)$ & $1(16,7)$ \\
Hipotireoidismo, $\mathrm{n}(\%)$ & $1(16,7)$ \\
Acesso femoral, $\mathrm{n}(\%)$ & $5(83,3)$ \\
Tamanho do aneurisma da artéria renal, $\mathrm{cm}$ & $3,2 \pm 1,1$ \\
Fibrodisplasia renal, $\mathrm{n}(\%)$ & $5(83,3)$ \\
\hline
\end{tabular}

As técnicas usadas para a correção endovascular do AAR estão descritas na tabela 2. Foram realizados quatro tratamentos endovasculares pela técnica de re- modelamento, com o uso de stent expansível por balão Palmaz ${ }^{\circledR}$ Blue $^{\circledR} 5$ ou $6 \mathrm{~mm}$ x 15 ou $18 \mathrm{~mm}$, associado ao uso de micromolas de $0,018^{\prime}$ AZUR ${ }^{\circledR}$. A liberação das molas foi realizada após cateterização do saco aneurismático com cateter diagnóstico $4 \mathrm{~F}$ ou microcateter Progreat $^{\circledR}$ 2,4 F, navegados por fio-guia 0,014 x $180 \mathrm{~cm}$ (Figura 4). Não foram observadas dissecções de artéria renal, perfurações de parênquima renal ou fístulas arteriovenosas no período periprocedimento.

Uma paciente apresentou aneurisma fusiforme tipo II na saída de um grande ramo troncular renal, e o tratamento escolhido foi a endoprótese Multilayer $^{\circledR}$, implantada com sucesso. No segundo dia de pós-operatório, ela evoluiu com dor em região lombar direita associada à hematúria macroscópica. A TC renal demostrou isquemia de todo o polo renal superior. Apesar do tratamento com analgésicos e opioides, a paciente evoluiu com dor incontrolável, sendo optado pela realização de nefrectomia total. Atualmente, a paciente encontra-se em acompanhamento ambulatorial, sem dor e sem deterioração da função renal (Figura 5).

Outra paciente apresentou aneurisma fusiforme tipo III em artéria renal segmentar distal, na qual foi 
utilizada a técnica de micronavegação com embolização superseletiva do ramo nutridor com uso de Histoacryl $^{\circledR}$. A paciente evoluiu sem queixas de dor lombar, hematúria ou piora da função renal no pós-operatório. Os controles ultrassonográficos demostraram exclusão do aneurisma renal.

Durante o seguimento de 1 ano (5 a 21 meses), não se observou endoleak ou retorno do fluxo ao saco aneurismático dos AARs tratados (Figura 6). A perviedade da artéria renal em 1 ano foi de $83,3 \%$. Não ocorreram óbitos, reintervenções ou nefrectomias. Nas cinco pacientes que apresentavam hipertensão arterial sistêmica, houve melhor controle pressórico, havendo diminuição das medicações anti-hipertensivas no pós-operatório.

\section{DISCUSSÃO}

AAR são raros. A prevalência em estudos de autópsia é de $0,01 \%$, porém, em pacientes selecionados para estudo arteriográfico renal, sua prevalência pode chegar a 0,3 a $1 \%$. São predominantes no sexo feminino, por sua forte associação com a fibrodisplasia muscular renal. ${ }^{8,13}$ Dentre os pacientes do United States Registry or Fibromuscular Dysplasia, 91\% eram mulheres e

TABELA 2

Detalhes técnicos das correções endovasculares de aneurismas de artéria renal

\begin{tabular}{lccc}
\hline Localização dos aneurismas & $\mathbf{n}(\%)$ & Características dos aneurismas de artéria renal & Técnica escolhida \\
\hline Zona 2 & $5(83,3)$ & $\begin{array}{c}\text { Sacular com colo largo }(\mathrm{n}=4) \\
\text { Fusiforme em bifurcação arterial }(\mathrm{n}=1)\end{array}$ & $\begin{array}{c}\text { Remodelamento com stent e molas } \\
\text { Endoprótese Multilayer }\end{array}$ \\
Zona 3 & $1(16,7)$ & Fusiforme & Embolização superseletiva com Histoacryl ${ }^{\circledR}$ \\
\hline
\end{tabular}
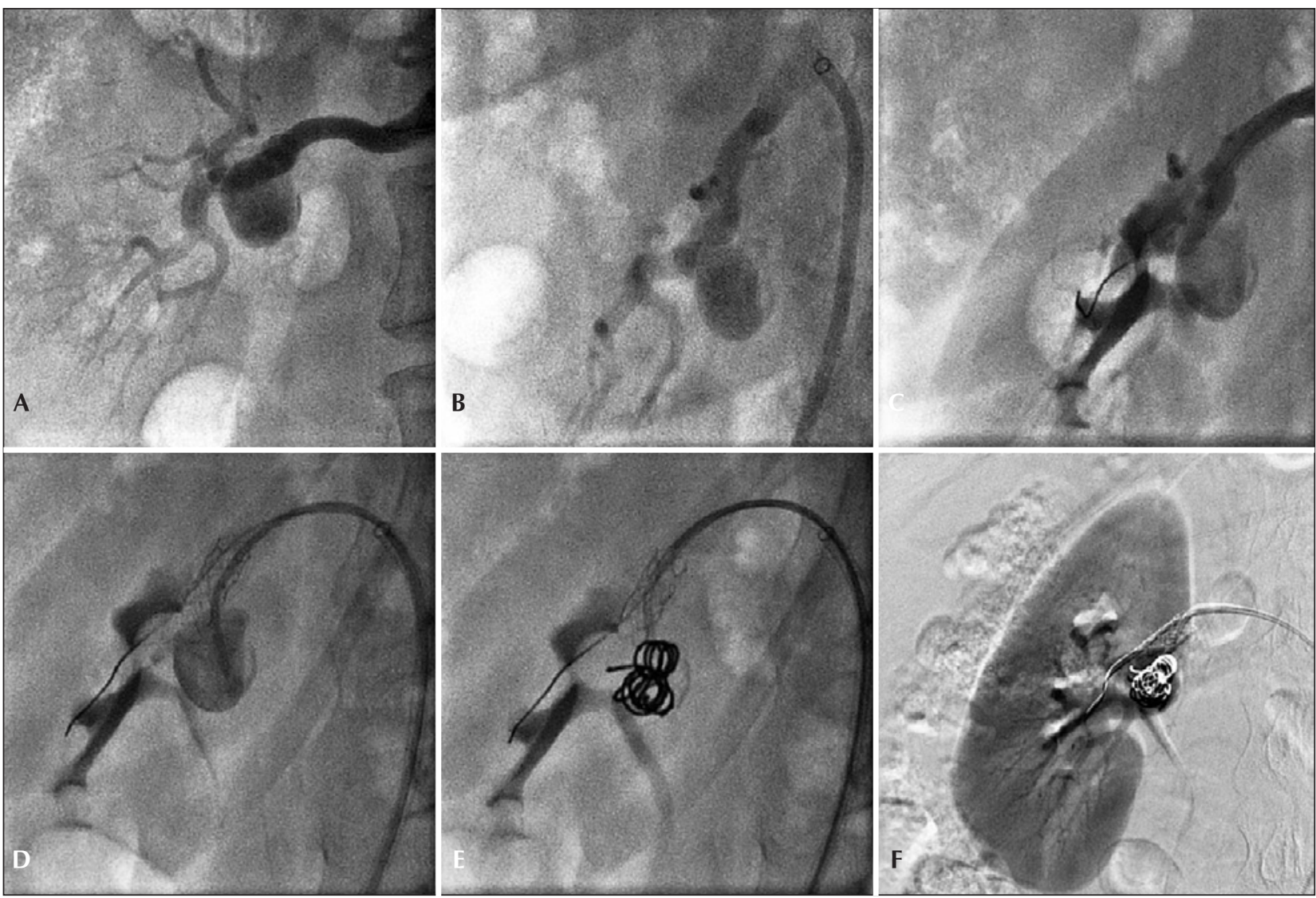

Figura 4. Passo a passo da técnica de remodelamento para o tratamento do aneurisma de artéria renal tipo II com uso de cateter 4 F. (A) Arteriografia de artéria renal direita. (B) Incidência de trabalho para visualização da artéria renal principal distal. (C) Implante de stent expansível por balão no colo aneurismático. (D) Cateterização do saco aneurismático com cateter JR $4 \mathrm{~F}$ entre as malhas do stent. (E) Liberação de molas 0,035 em saco aneurismático. (F) Arteriografia renal final. 
5,6\% apresentavam AAR. ${ }^{14} \mathrm{Em}$ nosso estudo, todos os pacientes eram do sexo feminino e cinco deles $(83,3 \%)$ apresentavam fibrodisplasia da artéria renal.

Sua história natural ainda é pouco conhecida. Sabe-se que os AARs podem ocorrer devido a trauma, infecção, arterites como a PAN, doença de Kawasaki, doença aterosclerótica ou displasias vasculares (fibrodisplasia da artéria renal ou síndrome de Ehlers-Danlos). A ruptura é um evento raro, com incidência relatada de 5 a $10 \%$, porém está associada a taxas de mortalidade de até $80 \%$. Dentre os três tipos de AAR, o tipo III apresenta maior risco de ruptura. ${ }^{2,8,9,15,16}$

Existe um consenso geral na literatura de que os AAR devem ser tratados quando encontrados os seguintes critérios: (1) AAR $>2 \mathrm{~cm}$ ou crescimento aneurismático documentado; (2) pacientes sintomáticos; (3) AAR com embolização distal documentada; (4) AAR em mulheres em idade fértil ou em gestação; (5) AAR associado à estenose significativa e à má perfusão renal. ${ }^{1,6,9,16} \mathrm{Em}$ nosso estudo, a maioria das pacientes teve indicação devido ao tamanho $>2 \mathrm{~cm}$, sendo que a maioria também era assintomática e ainda se encontrava em idade fértil.

Com os métodos atuais de imagens disponíveis, o planejamento pré-operatório detalhado é parte fundamental na determinação da técnica e na escolha dos materiais, para uma correção endovascular efetiva e um acompanhamento pós-operatório adequado. A TC permite avaliar a anatomia vascular renal, assim como as características dos aneurismas a serem tratados. Quando esse método diagnóstico não puder demonstrar todas as informações necessárias para o correto manuseio endovascular, a arteriografia pré-operatória deve ser realizada. Nos casos de aneurismas complexos, em que a melhor incidência para a visualização do colo aneurismático deve ser obtida, a angiografia rotacional, com reconstrução tridimensional, é essencial para a melhor compreensão da angioarquitetura vascular renal. ${ }^{9}$ Entretanto, no acompanhamento pós-operatório, a TC apresenta limites para a visualização adequada das molas e dos vazamentos, em razão dos artefatos gerados pela presença do metal, ${ }^{17}$ sendo o USG-D e a angiorressonância os métodos adequados para o seguimento desses pacientes. ${ }^{3,9}$

A experiência adquirida com o manejo endovascular das estenoses da artéria renal contribuiu para o aprendizado na abordagem endovascular dos AAR. ${ }^{18}$ Estudos com o tratamento endovascular desses aneurismas são restritos a casuísticas pequenas ou relatos de caso. ${ }^{19-23}$ Antoniou e Antoniou ${ }^{24}$ apresentaram revisão de 22 aneurismas tratados com uso de stents, com $91 \%$ de sucesso técnico e $27 \%$ de complicações menores, como a oclusão de pequenas artérias polares. Durante o seguimento de 8 meses, foi relatada uma reestenose da artéria renal. Elaassar et al. ${ }^{19}$ relataram o tratamento de 13 pacientes, utilizando 3 técnicas diferentes: embolização com molas $(n=8)$, emboli-
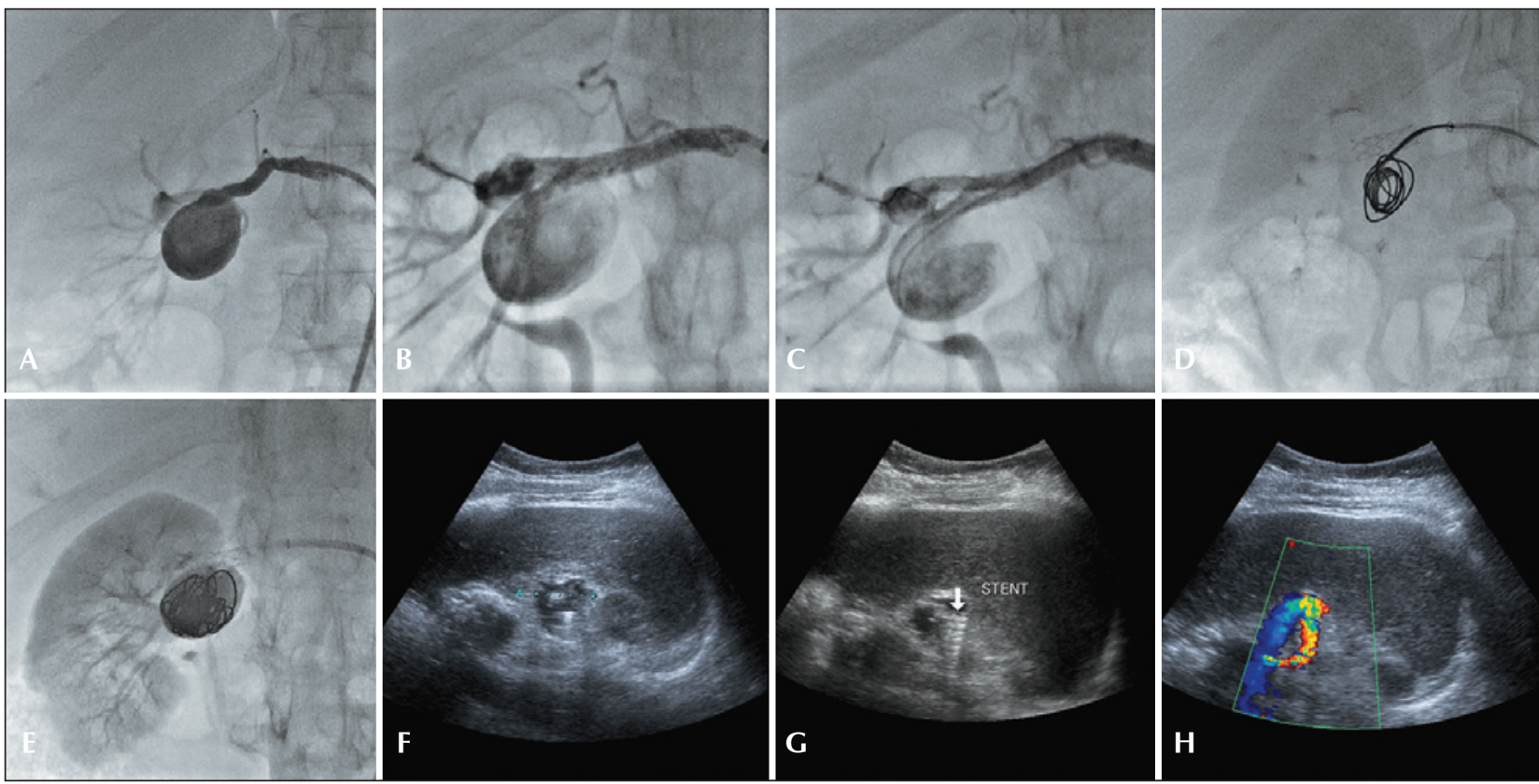

Figura 5. Tratamento de volumoso aneurisma de artéria renal tipo II pela técnica de remodelamento e acompanhamento pós-operatório com a ultrassonografia Doppler. (A) Arteriografia renal direita demonstrando volumoso aneurisma renal tipo II. (B) Incidência arteriográfica de trabalho. (C) Implante de stent expansível por balão e cateterização com cateter JR $4 \mathrm{~F}$ entre as malhas do stent. (D) Liberação de molas 0,035 no saco aneurismático. (E) Arteriografia final. (F) Acompanhamento pós-operatório com ultrassonografia em Modo-B demonstrando preenchimento total do saco aneurismático com molas. (G) Ultrassonografia Doppler em Modo B demonstrando a localização adequada do stent em colo de aneurisma renal. (H) Ultrassonografia Doppler em modo color demonstrando perviedade do stent e ausência de fluxo no saco aneurismático. 
zação com agentes líquidos $(\mathrm{n}=2)$ e uso de técnica de remodelamento com uso de stent e molas $(\mathrm{n}=3)$. Obtiveram sucesso técnico de $100 \%$ e taxa de oclusão de pequenos ramos renais de 23\%. Autores nacionais relataram o tratamento de 11 aneurismas de artérias renais complexos, tratados com técnicas de remodelamento em 8 pacientes e embolização em 3. Obtiveram sucesso técnico de $100 \%$, com um caso de oclusão de ramo num AAR tipo II tratado com apenas embolização por mola. Não observaram vazamentos ou recanalizações nos aneurismas tratados num seguimento de 32 meses. ${ }^{9}$

Em nosso estudo, obtivemos taxa de sucesso técnico de $100 \%$ e utilizamos três técnicas para o tratamento dos AARs, a depender da localização e da anatomia. Devido à predominância do AAR tipo II (83,3\%), utilizamos a técnica de remodelamento com uso de stent e molas em quatro casos, não se observando oclusões de ramos renais ou vazamentos com essa técnica. Em um caso de AAR tipo III, foi utilizada a técnica de embolização com agente líquido, com oclusão total do aneurisma e do vaso nutridor, não se observando isquemia renal, dor ou hematúria macroscópica no pós-operatório. Em um dos casos do AAR do tipo II, optou-se pelo uso da endoprótese Multilayer ${ }^{\circledR}$. Mesmo tendo sido implantada no local desejado, a paciente evoluiu com isquemia de todo o polo superior renal e dor incontrolável, sendo optado pela realização da nefrectomia. Isso gerou uma taxa de sucesso terapêutico de $83,3 \%$.
Rundback et al. ${ }^{10}$ Classificaram os aneurismas renais de acordo com sua localização angiográfica, ajudando a estabelecer as estratégias de tratamento. Nas lesões do tipo I, nas quais o AAR se origina da artéria renal principal, o tratamento com stent recoberto é suficiente para o adequado selamento do aneurisma, quando respeitado o limite de $15 \mathrm{~mm}$ entre o aneurisma e a origem da artéria renal, e deste até sua bifurcação. No tratamento desse tipo de aneurismas, dá-se preferência à utilização de stents recobertos com uma maior flexibilidade e uma liberação mais precisa, uma vez que pequenas migrações dos stents, causadas por tortuosidades da artéria renal ou pela imprecisão dos materiais escolhidos, podem ocluir ramos renais tronculares, com consequente isquemia renal. Embolizações seletivas com molas ou cola podem ser uma opção nesses tipos de aneurismas com colos pequenos e saculares. ${ }^{2,9,15}$ Em nossa casuística, não tivemos nenhum caso tratado de AAR tipo I.

Os AAR do tipo II eram antigamente tratados apenas por cirurgia convencional ou nefrectomia. Essas lesões constituem um desafio terapêutico para técnica endovascular pela dificuldade anatômica dessas lesões. Esses aneurismas compuseram a maior parte dos aneurismas tratados em nosso estudo. Nesses casos, a utilização de técnicas de remodelamento com uso de stent, associada ao uso de molas, ou de molas e cola, é fundamental para a preservação dos vasos nativos e o correto selamento do aneurisma. ${ }^{2,4,9,15,20}$
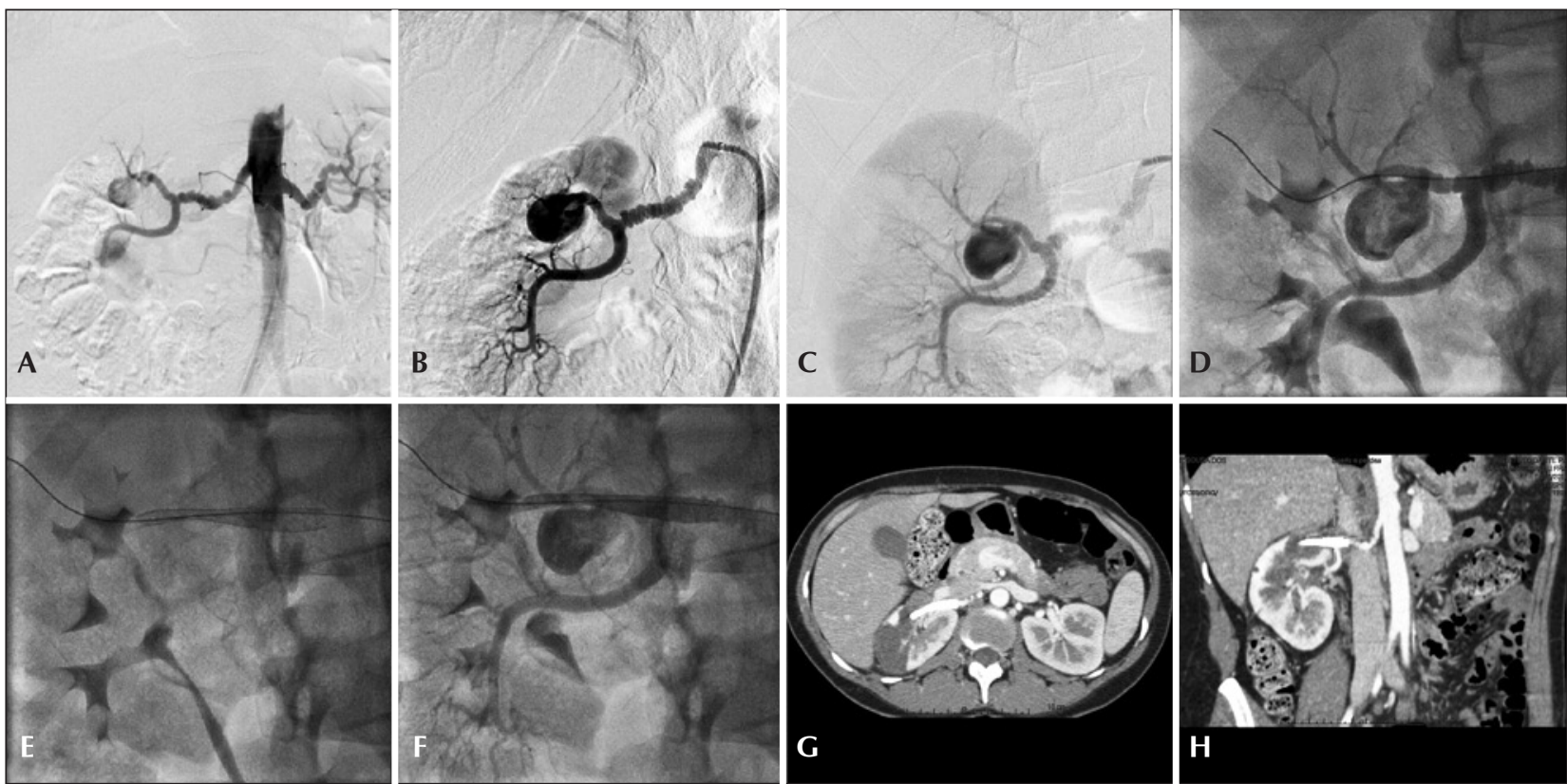

Figura 6. Tratamento do aneurisma de artéria renal do tipo II com uso de endoprótese Multilayer ${ }^{\circledR}$. (A) Aortografia demonstrando doença fibrodisplásica renal bilateral e aneurisma renal tipo II à direita. (B) Arteriografia renal seletiva à direita. (C) Incidência de trabalho com melhor visualização da bifurcação renal e sua relação com o colo do aneurisma. (D) Passagem de fio-guia 0,018 pela artéria renal principal distal. (E) Posicionamento e liberação da endoprótese Multilayer ${ }^{\circledR}$ sob artéria renal principal e no colo do aneurisma. (F) Arteriografia renal final. (G) Angiotomografia pós-operatória em corte axial demonstrando isquemia de polo renal superior. (H) Angiotomografia em corte coronal demonstrando perviedade de ramo renal troncular. 
Quanto aos aneurismas do tipo III, que emergem a partir de pequenas artérias segmentares e suprem uma pequena parte do parênquima renal, podem ser embolizadas com a oclusão do vaso nutridor. Agentes líquidos, como a cola e o Onyx ${ }^{\circledR}$ (Ev3 Inc., Plymouth, Estados Unidos), podem ser usados para esse propósito. Em nosso estudo, tivemos um caso de AAR do tipo III, que foi adequadamente embolizado com o uso de Hystoacril ${ }^{\circledR}$. 2,9,19,24

\section{Limitações do estudo}

Devido ao pequeno número de casos, ao grupo heterogêneo de pacientes e de tipos de procedimentos realizados, assim como pelo prazo curto de seguimento, a comparação entre as técnicas e a relação com seus desfechos limitam os resultados de nosso estudo. Por fim, a acurácia dos resultados pode ser afetada pela análise retrospectiva dos dados.

\section{CONCLUSÕES}

O tratamento endovascular dos aneurismas da artéria renal demonstrou ser tecnicamente viável e associado a baixos índices de morbimortalidade, no curto e médio prazos. O estudo detalhado da vascularização renal e da localização do aneurisma determina a escolha da técnica endovascular a ser utilizada. O aneurisma da artéria renal do tipo II foi a morfologia mais frequentemente encontrada e pode ser tratado com sucesso por técnicas de remodelamento, com o uso de stent e mola.

\section{CONFLITO DE INTERESSES}

Não há.

\section{FONTE DE FINANCIAMENTO}

Não há.

\section{REFERÊNCIAS}

1. Eskandari MK, Resnick SA. Aneurysms of the renal artery. Semin Vasc Surg. 2005;18(4):202-8.

2. Tsilimparis N, Reeves JG, Dayama A, Perez SD, Debus ES, Ricotta JJ 2nd. Endovascular vs open repair of renal artery aneurysms: outcomes of repair and long-term renal function. J Am Coll Surg. 2013;217(2):263-9.

3. Browne RF, Riordan EO, Roberts JA, Ridgway JP, Woodrow G, Gough $M$, et al. Renal artery aneurysms: diagnosis and surveillance with 3D contrast-enhanced magnetic resonance angiography. Eur Radiol. 2004;14(10):1807-12.

4. Goy JJ, Tinguely F, Poncioni L, Berger A, Stauffer JC. Aneurysm of the renal artery in a patient with the Marfan syndrome, treated by stenting and coils implantation. Catheter Cardiovasc Interv. 2007;69(5):701-3.

5. Soliman KB, Shawky Y, Abbas MM, Ammary M, Shaaban A. Ruptured renal artery aneurysm during pregnancy: a clinical dilemma. BMC Urol. 2006;6:22.
6. Malacrida G, Dalainas I, Medda M, Nano G, Inglese L. Endovascular treatment of a renal artery branch aneurysm. Cardiovasc Intervent Radiol. 2007;30(1):118-20.

7. Vallina-Victorero Vazquez MJ, Vaquero Lorenzo F, Salgado AA, Ramos Gallo MJ, Vicente Santiago M, Lojo Rocamonde IM, et al. Endovascular treatment of splenic and renal aneurysms. Ann Vasc Surg. 2009;23(2):258.e13-7.

8. Henke PK, Cardneau JD, Welling TH 3rd, Upchurch GR Jr, Wakefield TW, Jacobs LA, et al. Renal artery aneurysms: a 35year clinical experience with 252 aneurysms in 168 patients. Ann Surg. 2001;234(4):454-62.

9. Abath C, Andrade G, Cavalcanti D, Brito N, Marques R. Complex renal artery aneurysms: liquids or coils? Tech Vasc Interv Radiol. 2007;10(4):299-307.

10. Rundback JH, Rizvi A, Rozenblit GN, Poplausky M, Maddineni $S$, Crea G, et al. Percutaneous stent-graft management of renal artery aneurysms. J Vasc Interv Radiol. 2000;11(9):1189-93.

11. Henry M, Benjelloun A, Henry I, Wheatley G. The multilayer flow modulator stent for the treatment of arterial aneurysms. J Cardiovasc Surg (Torino). 2013;54(6):763-83.

12. Meyer C, Verrel F, Weyer G, Wilhelm K. Endovascular management of complex renal artery aneurysms using the multilayer stent. Cardiovasc Intervent Radiol. 2011;34(3):637-41.

13. English WP, Pearce JD, Craven TE, Wilson DB, Edwards MS, Ayerdi J, et al. Surgical management of renal artery aneurysms. J Vasc Surg. 2004;40(1):53-60.

14. Olin JW, Froehlich J, Gu X, Bacharach JM, Eagle K, Gray BH, et al. The United States Registry or Fibromuscular Dysplasia: results in the first 447 patients. Circulation. 2012;125(25):3182-90.

15. Wajnberg E, Aquino D, Spilberg G. Experiência preliminar com o uso da técnica de "remodelagem de colo" para tratamento endovascular de aneurismas complexos da artéria renal. Radiol Bras. 2010;43(1):29-33.

16. Ufberg JW, McNeil B, Swisher L. Ruptured renal artery aneurysm: an uncommon cause of acute abdominal pain. J Emerg Med. 2003;25(1):35-8

17. Tulsyan N, Kashyap VS, Greenberg RK, Sarac TP, Clair DG Pierce $\mathrm{G}$, et al. The endovascular management of visceral artery aneurysms and pseudoaneurysms. J Vasc Surg. 2007;45(2):276-83.

18. Patel VI, Conrad MF, Kwolek CJ, LaMuraglia GM, Chung TK, Cambria RP. Renal artery revascularization: outcomes stratified by indication for intervention. J Vasc Surg. 2009;49(6):1480-9.

19. Elaassar O, Auriol J, Marquez R, Tall P, Rousseau H, Joffre $\mathrm{F}$. Endovascular techniques for the treatment of renal artery aneurysms. Cardiovasc Intervent Radiol. 2011;34(5):926-35.

20. Kitzing B, Vedelago J, Bajic N, Lai G, Waugh R. Stent-assisted coil embolization of a wide-necked renal artery aneurysm. J Radiol Case Rep. 2010;4(4):20-4.

21. Xiong J, Guo W, Liu X, Yin T, Jia X, Zhang M. Renal artery aneurysm treatment with stent plus coil embolization. Ann Vasc Surg. 2010;24(5):695.e1-3.

22. Menegolo M, Frigatti $P$, Ferretto $L$, Antonello M, Grego F. Stent graft exclusion of a renal artery aneurysm at hilum in a case with complex anatomy. Perspect Vasc Surg Endovasc Ther. 2009;21(4):240-3.

23. Somarouthu B, Rabinov J, Waichi W, Kalva SP. Stent-assisted coil embolization of an intraparenchymal renal artery aneurysm in a patient with neurofibromatosis. Vasc Endovascular Surg. 2011;45(4):368-71.

24. Antoniou G, Antoniou S. Endovascular stent graft repair of renal artery aneurysms. Int Angiol. 2011;30(5):481-7. 